\title{
Development of Geopolymers for Catalyst Support Applications
}

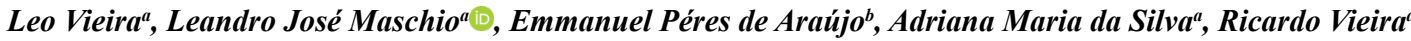 \\ ${ }^{a}$ Laboratório Associado de Combustão e Propulsão, Instituto Nacional de Pesquisas Espaciais, \\ Cachoeira Paulista, SP, Brasil \\ ${ }^{b}$ Departamento de Engenharia de Materiais, Escola de Engenharia de Lorena, Universidade de São \\ Paulo, Lorena, SP, Brasil
}

Received: November 29, 2018; Revised: May 20, 2019; Accepted: October 16, 2019

\begin{abstract}
A geopolymer with high specific surface area was synthesized from metakaolin, amorphous silica and $\mathrm{KOH}$, in the absence of foaming or saponification agents. The mixture modelling technique was selected for this study among the Design of Experiments tools, using surface area and total pores volume as response variables. $\mathrm{The} \mathrm{Si} / \mathrm{K}=2.46$ and $\mathrm{Si} / \mathrm{Al}=1.37 \mathrm{ratios}$ lead to the optimal experimental conditions, allowing the formation of a geopolymer having a specific surface area of $75 \mathrm{~m}^{2} / \mathrm{g}$ and total pores volume of $0.28 \mathrm{~cm}^{3} / \mathrm{g}$. The pores had a bimodal pore size distribution (7 and $\left.20 \mathrm{~nm}\right)$. In spite of its amorphous nature, this structure is similar to zeolites in terms of ion exchange and metal ions accommodation. Therefore, this study envisages its application as a catalyst support.
\end{abstract}

Keywords: Geopolymers, catalyst support, metakaolin, design of experiments.

\section{Introduction}

Geopolymers are inorganic materials produced by the activation of aluminium silicate in a high $\mathrm{pH}$ medium, which exhibit morphological and physicochemical properties suitable to catalytic support applications. They are also known as polysialates due to their structure based on tetrahedron chains, namely, $\mathrm{SiO}_{4}^{+}$and $\mathrm{AlO}_{4}^{+}$, interconnected by a shared oxygen atom $^{1}$. The characteristics of the geopolymer depend on the sources of the raw materials and $\mathrm{Si} / \mathrm{Al}$ molar ratio, as well as on the nature of the activators and the water content ${ }^{2,3}$. The main sources of aluminium silicates are kaolinite, metakaolin or fly ashes and the most frequently employed activators are $\mathrm{NaOH}$ and $\mathrm{KOH}^{4}$. The silicates previously dissolved in the alkaline solution provide the self-polymerizing species required to react with the tetrahedrons ${ }^{5}$. The mechanism of geopolymerization has been studied by Duxson et al. (2007). The authors have suggested a simplified mechanism comprising simultaneous solubilisation/polycondensation ${ }^{6}$. Initially, the solid grains of the aluminium silicate source are solubilised in the alkaline solution, yielding aluminate and silicate species, most probably in monomeric form. The super saturation of this solution results in a gel, linked by polycondensation to oligomers in the aqueous phase, whose size depends on the compensating cation. As polycondensation proceeds, an internal rearrangement takes place, leading to the formation of a three-dimensional net made of aluminates and silicates.

Geopolymers, also called inorganic polymers, appear as promising materials from the standpoint of mesoporous inorganic structures due to their combination of physical properties such as high porosity, mechanical resistance,

*e-mail: ricardo.vieira@inpe.br thermal stability and chemical inertness ${ }^{4}$. The pore structure is related to both the chemical nature and size of polysialates and to the interaction among the several phases in the material ${ }^{7,8}$. The porosity degree of geopolymers can reach values as high as $60 \mathrm{vol} . \%$, affecting drastically the thermal resistance. Nevertheless, the appropriate choice of $\mathrm{Si} / \mathrm{Al}$ ratio allows controlling the porosity in order to reach desirable thermal resistance values ${ }^{4}$. As a matter of fact, Sabbatini et al. (2017) demonstrated that the cation nature ( $\mathrm{Na}$ or $\mathrm{K}$ ) and the molar concentration of the alkaline medium $(>16$ $\mathrm{mol} / \mathrm{L}$ ) are critical parameters to the thermal resistance of the geopolymer ${ }^{9}$. Moreover, the silicon molar concentration ( $>39 \mathrm{~mol} / \mathrm{L}$ ) and the molar ratio between the alkaline metal and aluminium $(\mathrm{M} / \mathrm{Al}>0.65)$ are equally relevant to the porosity and, as a consequence, to the thermal resistance of the geopolymer. The mechanical resistance was also affected by the $\mathrm{Si}$ and $\mathrm{Al}$ content, with the highest value (6 $\mathrm{MPa})$ obtained at $\mathrm{Si} / \mathrm{Al}=1.75$. As for the structure of metakaolin-based geopolymers, it was demonstrated that all of the samples exhibited bulk instability in meso and macro scale, regardless of their molar ratio ${ }^{10}$.

As already mentioned, the physicochemical properties of geopolymer-based materials are also suitable for catalytic applications ${ }^{11}$. Foremost, their structure is comparable to zeolites, a well-known catalyst support, in terms of ion exchange and accommodation of metal ions, although geopolymers feature an amorphous morphology ${ }^{11}$. Undoubtedly, the great advantage related to geopolymers as catalyst supports relies on the thermal stability under demanding conditions. This behaviour has been explained by the strong interaction metal/support, preventing the metallic sintering under high temperatures and thus increasing the catalyst lifecycle ${ }^{12}$. 
Additionally, the acid-basic properties of geopolymers also provide stability to the catalyst toward carbon deposition, as verified under ethanol conversion ${ }^{12}$. Therefore, the activity of geopolymer-based catalysts has been evaluated in important industrial catalytic reactions with excellent performance, such as the selective catalytic reduction of $\mathrm{NO}_{\mathrm{x}}{ }^{13}$, total oxidation of volatile hydrocarbons (VOX) ${ }^{13}$ and biodiesel production ${ }^{14}$.

It is important to emphasize that the support may act solely as a physical frame to the active agent in the reaction, i.e., the catalyst, or it can play an active role in the process ${ }^{15,16}$. In both cases, the support must exhibit some characteristics to make it useful as a structure to the active phase, such as: (i) to yield a high exposed surface for the active agent; (ii) to enhance the stability of the catalytic material, by keeping the active phase particles highly dispersed; (iii) to increase the activity of the catalyst by providing a porous structure, allowing the access of the reactants to the active sites inside the porous channel and diffusion of the products from them; (iv) to dissipate promptly the heat from the reaction sites, thus avoiding the formation of hot spots and possible sintering of the active phase $\mathrm{e}^{11,15,16}$. Briefly, the catalyst support should yield a high specific surface area, enhanced porosity, compatible mechanical resistance, thermal conductivity, good chemical stability and moldability.

Therefore, this study applied the mixture modelling technique aiming at bringing more insight on the composition role of geopolymers on their specific surface area and total pores volume. Among the Design of Experiments (DoE) set of tools, the mixture approach was selected due to the nature of the problem under scrutiny, i.e., the controllable variables are not independent.

\section{Experimental}

\subsection{Preparation of the Materials}

Potassium hydroxide (Sigma-Aldrich, P1767) diluted into $30 \mathrm{~mL}$ of water was the activator used in all of the samples studied. Silica gel (Davicat) ground to the $125 \mu \mathrm{m}$ range was added to the potassium hydroxide solution, in order to adjust the $\mathrm{Si} / \mathrm{Al}$ ratio. The source of the aluminium silicate was metakaolin, produced through thermal treatment of the raw kaolin (Sigma-Aldrich K7375) at $973 \mathrm{~K}$ for $60 \mathrm{~min}$. The heating rate was $2 \mathrm{~K} / \mathrm{min}$. This procedure was suggested by KenneDiffo et al. (2015), who emphasized the importance of a slow heating rate to attain a faster polymerization velocity and a higher mechanical resistance ${ }^{17}$.

The chemical composition of the metakaolin was determined through the XRF technique, using a PANalytical equipment model Axios MAX. The metakaolin composition is described in Table 1.

\subsection{Preparation of the Samples}

All the geopolymers samples were synthetized keeping the water content constant ( $30 \mathrm{wt} \%$ ) while a different amount of $\mathrm{Si}$ was added in order to vary the $\mathrm{Si} / \mathrm{Al}$ molar ratio. According to the DoE mixture design approach, the solid was treated as a three-component mixture where the sum of the mass fractions added up to $100 \%$ throughout the whole experimental region. The objective was to investigate the role of $\mathrm{KOH}, \mathrm{Si} / \mathrm{Al}$ molar ratio over the specific surface area and the total pores volume of the final material.

The composition range (wt.\%) for each of the solids was set taking into account the simultaneous need for the three materials in order to produce a feasible geopolymer and the choice of metakaolin as a raw material. Metakaolin is formed by both aluminium and silicon oxides. Hence, the $\mathrm{Si} / \mathrm{Al}$ minimum ratio in the mixture is defined by the metakaolin composition. In order to increase this ratio, silica was added up to the desired superior limit. Therefore, the concentration ranges of the solids was set to be $0.12 \leq \mathrm{C}_{\mathrm{KOH}} \leq 0.30,0.42 \leq \mathrm{C}_{\mathrm{SiO} 2} \leq 0.60$ and $0.20 \leq \mathrm{C}_{\mathrm{Al} 2 \mathrm{O} 3} \leq 0.32$. The ternary diagram presented in Figure 1 depicts the region of interest in this study, which is defined by a hexagon, whose sides are delimited by the upper and lower concentration limits. The specific surface area and the total pores volume can then be examined within an experimental range comprising $\mathrm{Si} / \mathrm{Al}$ and $\mathrm{Si} / \mathrm{K}$ molar ratios, respectively, from 1.1 to 2.5 and 1.3 to 4.5 .

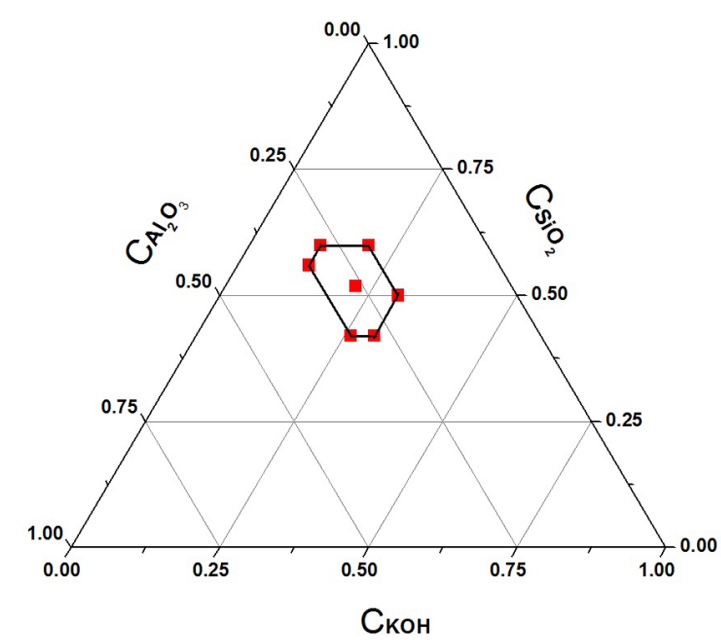

Figure 1. Three-component mixture design in terms of the real values.

The mixture contents in terms of pseudo-components, denoted by $X$, were calculated according to Equation (1):

$$
X_{i}=\frac{C_{i}-a_{i}}{1-\sum_{i=1}^{q} a_{i}}
$$

where $q$ is the number of components and $a_{i}$ is the lower limit of the $i^{\text {th }}$ component.

Table 1. Chemical composition of Metakaolin

\begin{tabular}{lcccccccc}
\hline Compound & $\mathrm{SiO}_{2}$ & $\mathrm{Al}_{2} \mathbf{O}_{3}$ & $\mathbf{T i O}_{2}$ & $\mathbf{F e}_{2} \mathbf{O}_{3}$ & $\mathbf{S O}_{3}$ & $\mathbf{P}_{2} \mathbf{O}_{5}$ & $\mathbf{K}_{2} \mathbf{O}$ & $\mathbf{C a O}$ \\
\hline Content $(\%)$ & 53.51 & 43.03 & 1.53 & 0.98 & 0.41 & 0.22 & 0.18 & 0.14 \\
\hline
\end{tabular}


Figure 2 shows the ternary diagram in terms of pseudocomponents. The fitting of a quadratic model can be performed with seven experimental points, defined by the vertices of the hexagon and its centroid $(\mathrm{C})$. This last point was run in a triplicate so that the experimental error can be computed and the quality of the fitted model can be assessed. From the seven compositions initially designed, all resulted in a homogeneous mixture with the aforementioned water content, except for point $\mathrm{nr} 5$. Thus, it was replaced by point $\mathrm{nr} 5$,' with a composition defined as the average of points 4 and 5 .

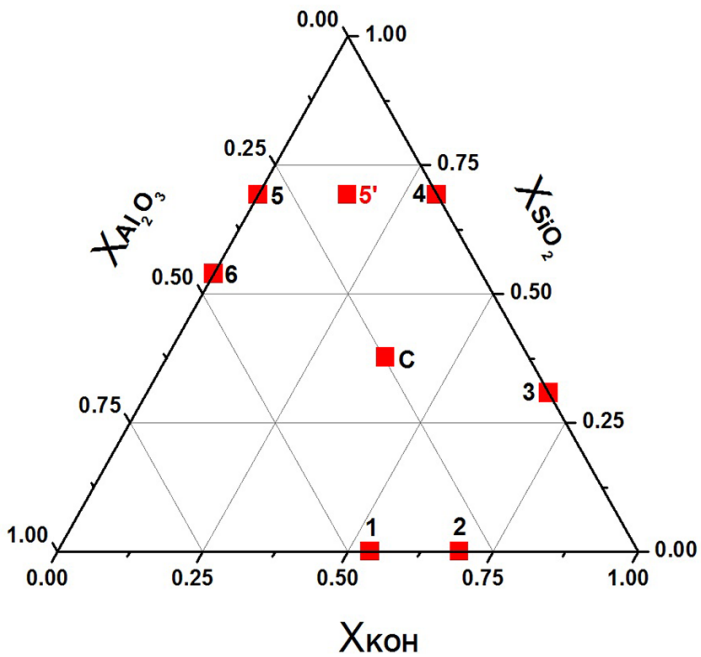

Figure 2. Three-component mixture design in terms of pseudocomponents.

All of the geopolymers samples were prepared according to a flexible protocol, spanning the entire experimental domain. The procedure started with the dissolution of the $\mathrm{KOH}$ aliquot in $30 \mathrm{~mL}$ of distilled water. After cooling down to circa $298 \mathrm{~K}$, the silica was slowly poured under constant rotation of $250 \mathrm{rpm}$, provided by a mechanical stirrer (IKA RW20). At last, the metakaolin was slowly added to the solution under stirring. In this step, it was not possible to standardize either the mixing time or the rotation, as long as the rheology of the experimental points was quite variable. Hence, these factors were adjusted dynamically. This last operation is the primer for the polymerization process, which proceeds after the pouring of the geopolymer dough into a cylindrical die, $3 \mathrm{~cm}$ internal diameter and $4 \mathrm{~cm}$ high.

The cast samples were kept for $24 \mathrm{~h}$ at around $298 \mathrm{~K}$. Subsequently, they were dried in a kiln, without atmosphere control, at $313 \mathrm{~K}$ for $24 \mathrm{~h}$ and then at $353 \mathrm{~K}$ for another $24 \mathrm{~h}$. Finally, the samples were calcined at $673 \mathrm{~K}$ for 60 minutes. The heating rate in this last step was $1 \mathrm{~K} / \mathrm{min}$.

Table 2 presents the compositions of the geopolymers in terms of the real values $(\mathrm{C} i)$ and pseudo-components $(X i)$, as well as the amounts $(m)$ of $\mathrm{KOH}$, metakaolin, and silicate used in each sample.

\subsection{Characterization of the Samples}

The parameters of interest in the samples were the specific surface area and the total pores volume. The specific surface area and the total pore volume were determined by $\mathrm{N}_{2}$ physissorption at $77 \mathrm{~K}$, employing a Micromeritics equipment model ASAP 2020C. The specific surface area and total pores volume were determined by applying the BET and BJH formalism to the experimental data, respectively.

\section{Results and Discussion}

The mixture design approach differs from the other $\mathrm{DoE}$ techniques because the response variables depend only on the components proportions (weight fractions) and are not affected by the total amount of the mixture. Therefore, this technique is suitable to study problems comprising two or more combined materials.

Table 3 presents the experimental matrix, including the proportions of each component, as well as the experimental results for the specific surface area and the total pores volume, generated by the seven different treatment combinations. The centroid was run in triplicate.

Table 2. Composition of the geopolymers in terms of real values and of pseudo-components.

\begin{tabular}{llllllllll}
\hline Exp. & $\boldsymbol{C}_{\text {КОН }}$ & $\boldsymbol{C}_{\text {SiO2 }}$ & $\boldsymbol{C}_{\text {Al2O3 }}$ & $\boldsymbol{X}_{\text {KOH }}$ & $\boldsymbol{X}_{\text {SiO2 }}$ & $\boldsymbol{X}_{\text {Al2O3 }}$ & $\boldsymbol{m}_{\text {KOH }}(\boldsymbol{g})$ & $\boldsymbol{m}_{\text {Silicate }}(\boldsymbol{g})$ & $\boldsymbol{m}_{\text {Metakaolin }}(\boldsymbol{g})$ \\
\hline 1 & 0.26 & 0.42 & 0.32 & 0.54 & 0.00 & 0.46 & 19.38 & 1.74 & 48.88 \\
2 & 0.30 & 0.42 & 0.28 & 0.69 & 0.00 & 0.31 & 22.04 & 5.19 & 42.77 \\
3 & 0.30 & 0.50 & 0.20 & 0.69 & 0.31 & 0.00 & 21.74 & 17.71 & 30.55 \\
4 & 0.20 & 0.60 & 0.20 & 0.31 & 0.69 & 0.00 & 14.74 & 24.71 & 27.55 \\
5 & 0.12 & 0.60 & 0.28 & 0.00 & 0.69 & 0.31 & 9.44 & 17.80 & 42.76 \\
5 & 0.16 & 0.60 & 0.24 & 0.15 & 0.69 & 0.15 & 12.09 & 21.26 & 36.65 \\
6 & 0.12 & 0.56 & 0.32 & 0.00 & 0.54 & 0.46 & 9.58 & 11.54 & 48.88 \\
$C$ & 0.22 & 0.52 & 0.26 & 0.38 & 0.38 & 0.25 & 16.25 & 13.45 & 40.30 \\
\hline
\end{tabular}


Table 3. Experimental design and corresponding responses.

\begin{tabular}{lccccc}
\hline Exp. & $\boldsymbol{X}_{\text {КОН }}$ & $\boldsymbol{X}_{\text {SiO2 }}$ & $\boldsymbol{X}_{\text {Al2O3 }}$ & $\boldsymbol{S}_{g}\left(\mathrm{~m}^{2} / g\right)$ & $\boldsymbol{V}_{\boldsymbol{p}}\left(\mathrm{cm}^{3} / \mathrm{g}\right)$ \\
\hline 1 & 0.54 & 0.00 & 0.46 & 18.5 & 0.1087 \\
2 & 0.69 & 0.00 & 0.31 & 9.0 & 0.0491 \\
3 & 0.69 & 0.31 & 0.00 & 3.0 & 0.0112 \\
4 & 0.31 & 0.69 & 0.00 & 0.1 & 0.0009 \\
5 & 0.15 & 0.69 & 0.15 & 0.7 & 0.0012 \\
6 & 0.00 & 0.54 & 0.46 & 44.5 & 0.1573 \\
$C$ & 0.38 & 0.38 & 0.24 & 52.0 & 0.1528 \\
$C$ & 0.38 & 0.38 & 0.24 & 60.0 & 0.1830 \\
$C$ & 0.38 & 0.38 & 0.24 & 58.0 & 0.1855 \\
\hline
\end{tabular}

Both the response variables were fitted to a quadratic model in order to understand their behaviours as a function of the mixture composition. The procedures are those outlined by Montgomery (2001) so that the fitting model is described by Equation (2), where $X$ stands for the pseudo-component independent variables ${ }^{18}$. Henceforth, $X_{1}, X_{2}$ and $X_{3}$ denote, respectively, $X_{\mathrm{KOH}} X_{\mathrm{SOO}_{2}}$ and $X_{\mathrm{AlOO}^{2}}$. All of the calculations were performed with the Scilab ${ }^{\circledR}$ software.

$\widehat{y}=b_{1}^{*} X_{1}+b_{2}^{*} X_{2}+b_{3}^{*} X_{3}+b_{12}^{*} X_{l} X_{2}+b_{13}^{*} X_{l} X_{3}+b_{23} X_{2} X_{3}$

Equation (3) presents the fitted quadratic model for the specific surface area:

$$
\begin{gathered}
S_{g}=-77.16 X_{1}-113.23 X_{2}-13.80 X_{3}+ \\
448.29 X_{1} X_{2}+286.87 X_{1} X_{3}+439.38 X_{2} X_{3}
\end{gathered}
$$

The Analysis of Variance (ANOVA) was then applied to assess the quality of the fitted model, i.e., how satisfactorily it describes the experimental domain. This is done by comparing the response variable fluctuations within the treatment combinations to the random errors inherently associated with any measuring procedure. Such a comparison allows the assessment of the regression significance, calculated by the ratio between the quadratic mean $(M S)$ of the regression and the quadratic mean of the residuals. This ratio $\left(F_{\text {calc. }}\right)$ was then compared to the tabulated $F$ critical values at the $95 \%$ confidence level $\left(F_{\text {cri.0.05 }}\right)$, considering the appropriate degrees of freedom, so that statistically significant values for $F_{\text {calc. }}$ must be greater than $F_{\text {cri. } 0.05}{ }^{19}$.

Table 4 outlines the ANOVA procedure for the model fitted to the specific surface area results.

The quadratic model fitted to the specific surface area data was significant, i.e., it was well adjusted to the experimental results. This conclusion was drawn from Table 4, where it is noticeable that $F_{\text {calc. }}=13.39>F_{\text {cri. } 0.05}=9.0$. Accordingly, the lack of fit test confirmed the excellent level of adherence of the experimental data to the fitted model, as long as $F_{\text {calc. }}=11.2<F_{\text {cri. } 0.05}=18.5$.

Likewise, the significance assessment of the fitted model coefficients stemmed from ANOVA. Significant interactions
Table 4. ANOVA calculations for the specific surface area data.

\begin{tabular}{lccccc}
\hline Source & $\boldsymbol{D F}$ & $\boldsymbol{S S}_{a d j .}$ & $\boldsymbol{M S}_{a d j .}$ & $\boldsymbol{F}_{\text {calc. }}$ & $\boldsymbol{F}_{c r i .0 .05}$ \\
\hline Regression & 5 & 5136.1 & 1027.2 & 13.39 & 9.0 \\
$X_{1}$ & 1 & 792.2 & 792.2 & 10.33 & 10.1 \\
$X_{2}$ & 1 & 1962.4 & 1962.4 & 25.59 & 10.1 \\
$X_{3}$ & 1 & 2.6 & 2.6 & 0.03 & 10.1 \\
$X_{1} X_{2}$ & 1 & 2104.7 & 2104.7 & 27.44 & 10.1 \\
$X_{1} X_{3}$ & 1 & 440.6 & 440.6 & 5.74 & 10.1 \\
$X_{2} X_{3}$ & 1 & 874.4 & 874.4 & 11.4 & 10.1 \\
Residue & 3 & 230.1 & 76.7 & & \\
Error & 2 & 34.7 & 17.4 & & \\
Lack offit & 1 & 195.4 & 195.4 & 11.2 & 18.5 \\
Total & 8 & 53662 & & & \\
\hline
\end{tabular}

between the $\mathrm{KOH}$ and $\mathrm{SiO}_{2}$ contents and between $\mathrm{SiO}_{2}$ and $\mathrm{Al}_{2} \mathrm{O}_{3}$ proportions were revealed by the data in Table 4 . On the other hand, no interaction was detected between the $\mathrm{KOH}$ and $\mathrm{Al}_{2} \mathrm{O}_{3}$ contents.

Figure 3 shows the contour plot of the fitted model (pseudo-components), revealing a region that maximizes the specific surface area, within the experimental domain investigated.

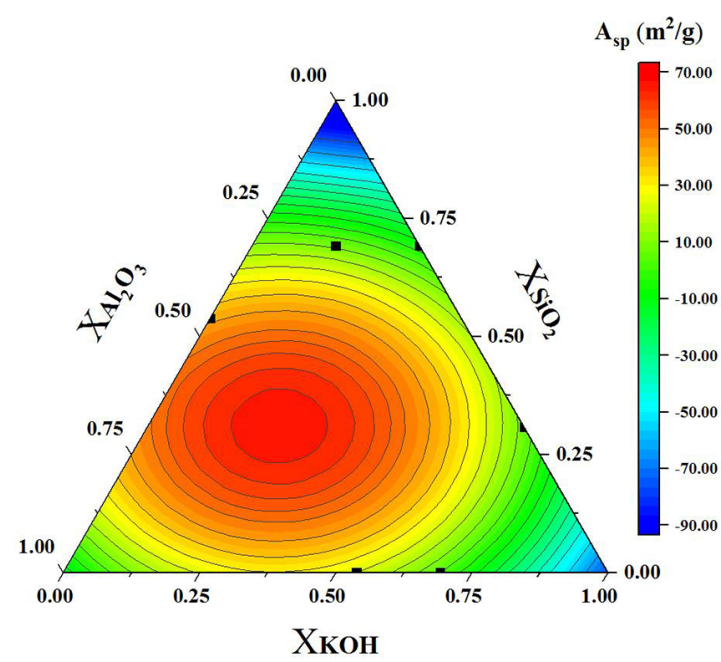

Figure 3. Contour plot of the specific surface area in terms of the investigated compositions.

The coordinates of the highest specific surface area point can be obtained through a partial derivation procedure of Equation (3), which should first be modified by the condition $X_{3}=1-X_{1}-X_{2}$. This modification is a required step because, due to the three-component mixture design approach adopted in this study, the problem has in fact two degrees of freedom. Equation (4) presents the modified specific surface area relationship:

$$
\begin{gathered}
S_{g}=-77.16 X_{1}-113.23 X_{2}-13.80\left(1-X_{1}-X_{2}\right)+ \\
448.29 X_{1} X_{2}+286.87 X_{1}\left(1-X_{1}-X_{2}\right)+ \\
439.38 X_{2}\left(1-X_{1} X_{2}\right)
\end{gathered}
$$


The partial derivatives of Equation (4), with respect to $X_{1}$ and $X_{2}$ provide an equation system that can be solved to yield the predicted composition of highest specific surface area. The results for the pseudo components $X_{1}, X_{2}$ and $X_{3}$, i.e., $X_{K O H} X_{S O_{2} 2}$ and $X_{A 12 O O}$, were $0.24,0.31$ and 0.45 , respectively.

The values for $C_{K O H}, C_{S^{2 O 2} 2}$ and $C_{A 12 O 3}$, calculated by means of Equation (1), are $0.19,0.50$ and 0.31 , respectively. Hence, it was possible to calculate the amounts needed for the production of $1 \mathrm{~kg}$ of geopolymer (Table 5), yielding a theoretical specific surface area of $66 \mathrm{~m}^{2} / \mathrm{g}$.

Table 5. Amounts of raw materials required to prepare $1 \mathrm{~kg}$ of the geopolymer.

\begin{tabular}{lcccc}
\hline & Metakaolin & Silicate & KOH & Water \\
\hline Mass $(\mathrm{g})$ & 473.5 & 82 & 144.5 & 300 \\
\hline
\end{tabular}

It is worthwhile noticing that the literature usually provides these amounts in an atomic molar ratio basis ${ }^{2,3,12,20}$, i.e., $(\mathrm{Si} / \mathrm{K})$ and $(\mathrm{Si} / \mathrm{Al})$. Therefore, regarding the data presented in Table 5, the molar ratios that provide the highest specific surface area, within the experimental domain studied, are $\mathrm{Si} / \mathrm{K}=2.46$ and $\mathrm{Si} / \mathrm{Al}=1.37$.

The pores volume, the second response variable of interest in this study, was investigated accordingly. Equation (5) shows the fitted quadratic model for this response, in terms of pseudo-components.

$$
\begin{aligned}
& V_{p}=-0.2278 X_{1}-0.3314 X_{2}+0.2001 X_{3}+ \\
& 1.3254 X_{1} X_{2}+0.6121 X_{1} X_{3}+0.9508 X_{2} X_{3}
\end{aligned}
$$

As before, the suitability of this model was assessed through ANOVA, which is depicted in Table 6. The quadratic model fitted to the pores volume data was significant, i.e., adjusted well to the experimental results. This conclusion is drawn from the values in Table 6, where one can notice that $F_{\text {calc. }}=13.99>F_{\text {cri. } 0.05}=9.0$. The lack of fit test has revealed no evidence of such a problem, as long as $F_{\text {calc. }}=4.24<F_{\text {cri.0.05 }}=18.5$. Hence, Equation (5) can be applied to predict the pores volume within the experimental domain.

Table 6. ANOVA calculations for the pores volume data.

\begin{tabular}{lccccc}
\hline Source & $\boldsymbol{D} \boldsymbol{F}$ & $\boldsymbol{S S}_{a d j .}$ & $\boldsymbol{M S}_{a d j .}$ & $\boldsymbol{F}_{\text {calc. }}$ & $\boldsymbol{F}_{c r i .0 .05}$ \\
\hline Regression & 5 & 0.0481 & 0.0096 & 13.99 & 9.0 \\
$X_{1}$ & 1 & 0.0069 & 0.0069 & 10.1 & 10.1 \\
$X_{2}$ & 1 & 0.0168 & 0.0168 & 24.48 & 10.1 \\
$X_{3}$ & 1 & 0.00054 & 0.00054 & 0.79 & 10.1 \\
$X_{1} X_{2}$ & 1 & 0.0184 & 0.0184 & 26.8 & 10.1 \\
$X_{1} X_{3}$ & 1 & 0.002 & 0.002 & 2.92 & 10.1 \\
$X_{2} X_{3}$ & 1 & 0.0041 & 0.0041 & 5.96 & 10.1 \\
Residue & 3 & 0.0021 & 0.00069 & & \\
Error & 2 & 0.00066 & 0.00033 & & \\
Lack of fit & 1 & 0.0014 & 0.0014 & 4.24 & 18.5 \\
Total & 8 & 0.0096 & 0.050126 & & \\
\hline
\end{tabular}

Once again, the significance assessment of the fitted model coefficients stems from ANOVA. The data in Table 6 shows significant interactions only between the $\mathrm{KOH}\left(X_{1}\right)$ and $\mathrm{SiO}_{2}$ $\left(X_{2}\right)$ proportions. No other significant interactions were detected.

The joint assessment of the significances depicted in Tables 4 and 6 reveals that the $\mathrm{Al}_{2} \mathrm{O}_{3}$ content $\left(\mathrm{X}_{3}\right.$ pseudocomponent $)$ is not significant by itself and presents a single significant interaction, i.e., $\mathrm{X}_{2} \mathrm{X}_{3}$, for the specific surface area. Bearing in mind that the nature of the problem under investigation does not allow an independent optimization of the responses and taking into account the higher importance of the surface area, $\mathrm{X}_{3}$ was not disregarded for the pores volume, as would be necessary if it were the only response of interest.

Figure 4 depicts the contour plot of the fitted model (pseudo-components), revealing a region of maxima outside the experimental domain investigated and non-coincident with the maxima region observed for the specific surface area. This stems from the fact that a high total pores volume does not necessarily translates into a high specific surface area. As an example, a certain material can exhibit a high total pores volume, where the pores with large diameter are predominant, thus reducing the specific area.

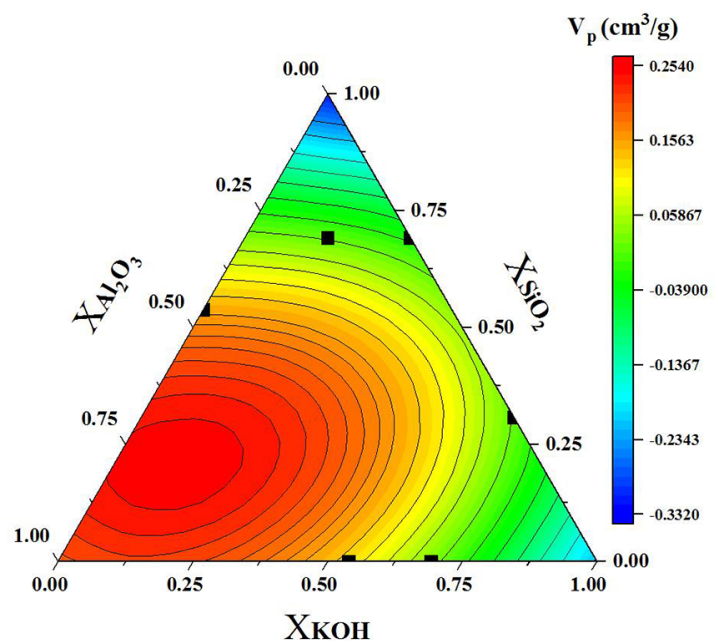

Figure 4. Contour plot of the pores volume in terms of the investigated geopolymer compositions.

Nevertheless, the aforementioned procedure for the calculation of the coordinates of the predicted highest total pores volume was applied, yielding for the pseudo components $X_{1}, X_{2}$ and $X_{3}$, i.e., $X_{\mathrm{KOH}}, X_{\mathrm{SiO}_{2}}$ and $X_{A 12 O 3}$, the values 0.11, 0.21 and 0.68 , respectively. Turning these results back into real variables, by means of Equation (1), yields the values for $C_{\mathrm{KOH}}, C_{\mathrm{SiO}_{2}}$ and $C_{\mathrm{Al2O} 3}$, respectively $0.15,0.47$ and 0.38 .

As stated above and illustrated in Figure 4, these coordinates are outside the experimental region studied. This fact led to an attempt to prepare the geopolymer with such a composition. Unfortunately, it was impossible to achieve an acceptable degree of homogeneity in this attempt, given the experimental constraint posed by the water content. Therefore, 
the expected dislocation of the geopolymer composition to the region of maximum total pores volume, by means of a simultaneous enhancement of the aluminium content and a reduction of the potassium amount, was not feasible. Considering this practical limitation and the prevalence of the specific surface area for the envisaged application of the geopolymer as a catalyst support, it was decided to adopt the best predicted composition obtained for the specific surface area, i.e., $X_{\mathrm{KOH}}=0.24, X_{\mathrm{SiO} 2}=0.31$ and $X_{A l 2 O 3}=0.45$. Thus, applying these values into Equation (5), a pores volume of $0.23 \mathrm{~cm}^{3} / \mathrm{g}$ is predicted.

The next obvious step in a DoE approach is to perform a confirmatory experiment. This was accomplished by following strictly the protocols of preparation and characterization of the samples described previously. As aforementioned, the primary interest on the specific surface area has led to the choice of its highest predicted result as the composition to be prepared, namely, $C_{\mathrm{KOH}}=0.19, C_{\mathrm{SiO} 2}=0.50$ and $C_{\mathrm{Al2O} 3}=0.31$. This geopolymer yielded a specific surface area of $75 \mathrm{~m}^{2} / \mathrm{g}$ and a total pores volume of $0.28 \mathrm{~cm}^{3} / \mathrm{g}$. These results, when compared to their theoretical predictions, respectively, $66 \mathrm{~m}^{2} / \mathrm{g}$ and $0.23 \mathrm{~cm}^{3} / \mathrm{g}$, show a satisfactory agreement, thus validating the fitted models.

Figure 5 shows the distribution of pores sizes for this optimized geopolymer. It is noticeable the nearly bimodal distribution, centered at $7 \mathrm{~nm}$ and $20 \mathrm{~nm}$. It is worth to say that the specific area of this material can also be increased using triglyceride oils for a reactive (saponification reaction)

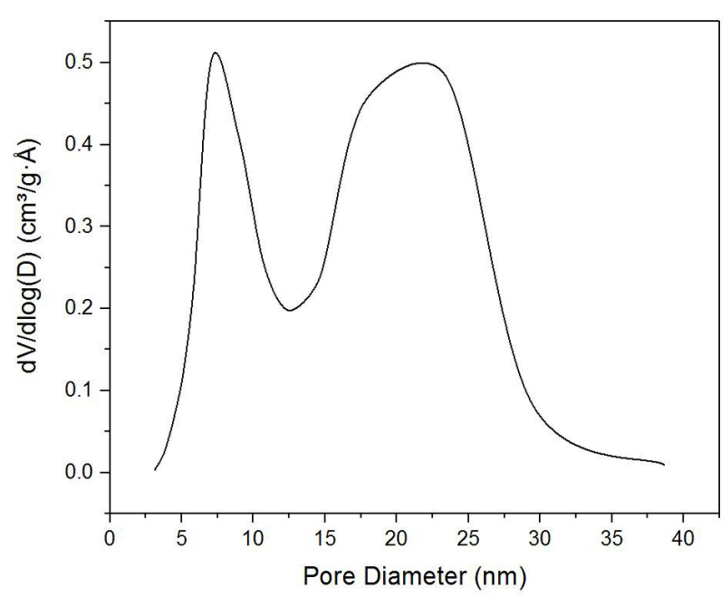

Figure 5. Distribution of pores sizes for the optimized geopolymer.

emulsion template ${ }^{21}$ or using hydrogen peroxide as foaming agent to control the porosity of the geopolymer ${ }^{22}$.

\section{Conclusions}

In this study, geopolymers were successfully prepared from mixtures containing $\mathrm{KOH}$, metakaolin, silicate and water, keeping the solids content at $70 \mathrm{wt} . \%$. The mixture design approach was used aiming at maximizing the specific surface area through the weight fractions of the raw materials, thus making foaming agents unnecessary in the production process of a catalyst support. The results showed that the geopolymer containing the molar ratios $\mathrm{Si} / \mathrm{K}=2.46$ and $\mathrm{Si} / \mathrm{Al}=1.37$ yield the highest specific surface area and pore volume, with values as high as $75 \mathrm{~m}^{2} / \mathrm{g}$ and $0.28 \mathrm{~cm}^{3} / \mathrm{g}$, respectively. The pores had a bimodal pore size distribution (primary and secondary average pore diameters are, respectively, 7 and 20 $\mathrm{nm})$, i.e. in the mesoporous range, which easily allows the diffusion of reactants and products, making this geopolymer a potential candidate for application in catalysis.

\section{Acknowledgments}

The authors acknowledge the partnership with the company Peróxidos do Brasil (Solvay Group) and FAPESP.

\section{References}

1. Davidovits J. Geopolymers inorganic polymeric new materials. Journal of Thermal Analysis. 1991;37:1633-1656.

2. Ozer I, Soyer-Uzun S. Relations between the structural characteristics and compressive strength in metakaolin based geopolymers with different molar Si/Al ratios. Ceramics International. 2015;41(8):10192-10198.

3. Novais RM, Ascensão G, Ferreira N, Seabra MP, Labrincha JA. Influence of water and aluminium powder content on the properties of waste-containing geopolymer foams. Ceramics International. 2018;44(6):6242-6249.

4. Bai C, Colombo P. Processing, properties and applications of highly porous geopolymers: A review. Ceramics International. 2018;44(14):16103-16118.

5. Xu H, Van Deventer JSJ. The effect of alkali metals on the formation of geopolymeric gels from alkali-feldspars. Colloids and Surfaces A: Physicochemical and Engineering Aspects. 2003;216(1-3):27-44

6. Duxson P, Fernandez-Jiménez A, Provis JL, Lukey GC, Palomo A, Van Deventer JSJ. Geopolymer technology: The current state of the art. Journal of Materials Science. 2007;42(9):2917-2933.

7. Duxson P, Provis JL, Lukey GC, Mallicoat SW, Kriven WM, Deventer JSJV. Understanding the relationship between geopolymer composition, microstructure and mechanical properties. Colloids and Surfaces A: Physicochemical and Engineering Aspects. 2005;269(1-3):47-58.

8. Duxson P, Provis JL, Lukey GC, Mallicoat SW, Kriven WM, Deventer JSJV. The effect of alkali and $\mathrm{Si} / \mathrm{Al}$ ratio on the development of mechanical properties of metakaolin-based geopolymers. Colloids and Surfaces A: Physicochemical and Engineering Aspects. 2007;292(1):8-20.

9. Sabbatini A, Vidal L, Pettinari C, Sobrados I, Rossignol S. Control of shaping and thermal resistance of metakaolin-based geopolymers. Materials and Design. 2017;116:374-385.

10. Lahoti M, Wong KK, Yang EH, Tan KH. Effects of Si/Al molar ratio on strength endurance and volume stability of metakaolin geopolymers subject to elevated temperature. Ceramics International. 2018;44(5):5726-5734. 
11. Sazama P, Bortnovsky O, Dedecek J, Tvaruzkova Z, Sobalík Z. Geopolymer based catalysts - New group of catalytic materials. Catalysis Today. 2011;164(1):92-99.

12. Candamano S, Frontera P, Macario A, Crea F, Nagy JB, Antonucci PL. Preparation and characterization of active Ni-supported catalyst for syngas production. Chemical Engineering Research and Design. 2015;96:78-86.

13. Zhang Z, Jiang Z, Shangguan W. Low-temperature catalysis for VOCs removal in technology and application: A state-of-the-art review. Catalysis Today. 2016;264:270-278.

14. Sharma S, Medpelli D, Chen S, Seo DK. Calcium-modified hierarchically porousaluminosilicate geopolymer as a highly efficient regenerable catalyst for biodiesel production. Royal Society of Chemistry Advances. 2015;5(80):65454-65461.

15. Ross JRH. Heterogeneous Catalysis: Fundamentals and Applications. Amsterdam: Elsevier; 2011.

16. Nørskov JK, Studt F, Abild-Pedersen F, Bligaard T. Fundamental Concepts in Heterogeneous Catalysis. New Jersey: Wiley; 2014.
17. Kenne BBD, Elimbi A, Cyr M, Manga JD, Tchakoute HK. Effect of the rate of calcination of kaolin on the properties of metakaolin-based geopolymers. Journal of Asian Ceramic Societies. 2015;3:130-138.

18. Montgomery D. Design and Analysis of Experiments. New York: John Wiley \& Sons; 2001.

19. Bezerra MA, Santelli RE, Oliveira EP, Villar LS, Escaleira LA. Response surface methodology (RSM) as a tool for optimization in analytical chemistry. Talanta. 2008;76(5):965-77.

20. Trindade ACC, Silva FA, Alcamand HA, Borges PHR. On the mechanical behavior of metakaolin based geopolymers under elevated temperatures. Materials Research. 2017;20(Suppl 2):265-272.

21. Medpelli D, Seo JM, Seo DK. Geopolymer with hierarchically meso-/macroporous structures from reactive emulsion templating. Journal of American Ceramic Society. 2014;97(1):70-73.

22. Hajimohammadi A, Ngo T, Mendis P, Nguyen T, Kashani A, Van Deventer JSJ. Pore characteristics in one-part mix geopolymers foamed by $\mathrm{H}_{2} \mathrm{O}_{2}$ : Theimpact of mix design. Materials and Design. 2017;130:381-391. 